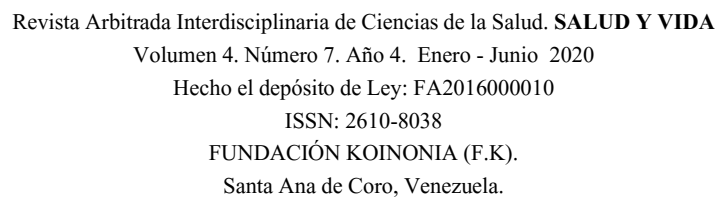

Aniervic Manuel Betancourt-Ruiz; Mayela Ysabel García

http://dx.doi.org/10.35381/s.v.v4i7.646

\title{
Factores asociados a la no adherencia del control prenatal en gestantes
}

\section{Factors associated with the non-adherence of prenatal control in gestants}

\author{
Aniervic Manuel Betancourt-Ruiz \\ aniervic@gmail.com \\ Hospital Universitario Dr. Alfredo Van Grieken, Santa Ana de Coro \\ Venezuela \\ https://orcid.org/0000-0001-5862-8640 \\ Mayela Ysabel García \\ dramayelag@gmail.com \\ Hospital Universitario Dr. Alfredo Van Grieken, Santa Ana de Coro \\ Venezuela \\ https://orcid.org/0000-0003-0526-8084
}

Recibido: 1 de octubre de 2019

Aprobado: 28 de noviembre de 2019

\section{RESUMEN}

El embarazo es un proceso fisiológico que inicia con la fecundación, para dar paso a la vida. El control prenatal consiste en una serie de consultas sucesivas que tienen como finalidad disminuir la morbi-mortalidad materno fetal. Objetivo: Determinar cuáles son los factores asociados a la no adherencia al control prenatal en gestantes adscritas al ambulatorio Manuel Navas Lizardo, Capatárida Municipio Buchivacoa en el lapso Agosto 2018 - Enero 2019. Metodología: Estudio descriptivo, corte transversal, de campo, no experimental cuya muestra fue el total de la población, abarcando 50 gestantes, obteniendo la información a través de una ficha de recolección de datos.. Conclusión: Entre los factores asociados a la no adherencia al control prenatal destacan: factor económico, transporte, distancia del centro de salud, filas de espera, asignación tardía de la cita y deficiente nivel de conocimiento acerca del control prenatal. Por lo que es necesario promover charlas o talleras por parte del personal de salud acerca de la importancia de asistir a consulta prenatal.

Descriptores: Embarazo; adherencia; control prenatal. 


\begin{abstract}
Pregnancy is a physiological process that begins with fertilization, to give way to life. Prenatal control consists of a series of successive consultations that aim to reduce maternal fetal morbidity and mortality. Objective: To determine what are the factors associated with non-adherence to prenatal control in pregnant women assigned to the Manuel Navas Lizardo outpatient clinic, Capatárida Municipality Buchivacoa in the period August 2018 - January 2019. Methodology: Descriptive study, cross-sectional, nonexperimental section whose Sample was the total population, covering 50 pregnant women, obtaining the information through a data collection form. Conclusion: Among the factors associated with non-adherence to prenatal control, the following stand out: economic factor, transportation, distance from the center of health, waiting lines, late appointment appointment and poor level of knowledge about prenatal control. Therefore, it is necessary to promote talks or heels on the part of health personnel about the importance of attending prenatal consultation.
\end{abstract}

Descriptors: Pregnancy; adherence; prenatal control.

\title{
INTRODUCCIÓN
}

El embarazo es un proceso fisiológico que inicia con la fecundación, donde se produce la unión del gameto femenino con el gameto masculino, sin embargo, existe una polémica sobre cuando comienza el embarazo, es así que, para la Organización Mundial de la Salud (OMS) el embarazo comienza cuando termina la implantación del embrión en el útero, ya que, en muchos casos, el óvulo es fecundado pero no llega a implantarse y el embarazo no comienza. La implantación es un proceso que comienza unos 5 o 6 días después de la fecundación y que consiste en la adherencia del blastocito a la pared del útero, cuando el blastocito atraviesa el endometrio e invade el estroma, luego la superficie del epitelio se cierra y se completa el proceso de nidación, comenzando entonces el embarazo. ${ }^{1}$

Entonces, para muchos científicos, la vida empieza en el momento de la fecundación y, por lo tanto, el embarazo comienza cuando un espermatozoide alcanza y atraviesa la membrana celular del óvulo, fusionándose los núcleos y compartiendo ambos su dotación genética para dar lugar a una célula huevo o cigoto, posteriormente la multiplicación celular del cigoto da lugar al embrión, de cuyo desarrollo deriva el individuo adulto. Así pues, fecundación y embarazo son contemporáneos y esa es la posición sostenida por 
las organizaciones Provida y por el Comité de Bioética de la Comisión de Familia de la Conferencia Episcopal Peruana. ${ }^{2}$

En 2007, el Comité de Aspectos Éticos de la Reproducción Humana y la Salud de las Mujeres de la Federación Internacional de Ginecología y Obstetricia (FIGO) definió al embarazo como la parte del proceso de la reproducción humana que comienza con la implantación del conceptus en la mujer. ${ }^{3}$ Así mismo después de la fecundación inicia el desarrollo prenatal, el cual se establece con la apertura de la 4ta semana: el embrión mide $4 \mathrm{~mm}$, pesa $1 \mathrm{~g}$, inicia el crecimiento incipiente de la cabeza, hay presencia de latido cardiaco; A las 8 semanas: mide aproximadamente $3 \mathrm{~cm}$ y pesa $3 \mathrm{~g}$, comienza el desarrollo de brazos y piernas, así como del cerebro y órganos internos, en la semana 12: Mide $10 \mathrm{~cm}$ y pesa $45 \mathrm{~g}$, desarrolla los párpados y movimiento de las extremidades, con 16 Semanas: mide $15 \mathrm{~cm}$ y pesa $180 \mathrm{~g}$, Se cubre de lanugo, el intestino comienza a llenarse de meconio, la piel es todavía muy fina, casi transparente. ${ }^{4}$

A partir de la semana 20: mide $18 \mathrm{~cm}$ y pesa $500 \mathrm{~g}$, crece el cabello, pestañas y cejas y desarrolla su sistema inmunitario; En la semana 24: mide $25 \mathrm{~cm}$ y pesa $1000 \mathrm{~g}$, la cara ya está completamente formada, la piel se cubre de vérnix caseoso, abre los ojos y se mueve mucho. La semana 28 es indispensable: talla $30 \mathrm{~cm}$ y pesa $1500 \mathrm{~g}$, inicia la maduración pulmonar, aumenta la grasa subcutánea. Con la llegada de la semana 32 : alcanza $35 \mathrm{~cm}$ y pesa $2500 \mathrm{~g}$, presenta engrosamiento de la piel, adquiriendo el tono rosáceo que tendrá definitivamente. Finalmente a las 36 semanas: Mide $50 \mathrm{~cm}$ y pesa aproximadamente $3000 \mathrm{~g}$. Los pulmones ya están completamente formados para la vida exterior, se cae el lanugo y la piel se estira. ${ }^{4}$

El diagnóstico se basa en la detección de signos y síntomas, siendo el ultrasonido una herramienta esencial, ya que permite detectar estructuras fetales que varían según la edad gestacional, como lo son saco gestacional y la actividad cardíaca fetal, la cual se evidencia a las 5ta semana como un latido regular con frecuencia entre 120 y 160 latidos por minuto, independiente del latido materno, sin embargo, la auscultación directa mediante el uso de estetoscopio fetal facilita la detección del latido cardíaco a partir de la semana 17-18 pero, en general, es en la semana 20 cuando adquiere un valor diagnóstico, sumado a la presencia de movimientos fetales activos constituyen los signos positivos de embarazo. ${ }^{5}$ 
Entre los signos probables se encuentra, la determinación de la Hormona Gonadotrófica Corionica Humana (HCG) en sangre siendo factible uno o dos días luego de la implantación del trofoblasto; es decir, unos siete a ocho días después de la fecundación, esta misma alcanza su pico sérico máximo alrededor de la semana 8-12 y luego desciende bruscamente hasta la semana 20, a partir de la cual se mantiene más o menos estable hasta el momento del parto, después del cual desaparece. El pico máximo es de $50.000 \mathrm{mUl} / \mathrm{cc}$ con un rango de hasta $150.000 \mathrm{mUl} / \mathrm{cc}$ Otras hormonas como son la adrenocorticotropina, la liberadora de hormona luteinizante y la progesterona son también útiles para el diagnóstico del embarazo. ${ }^{5}$

Por otra parte, todo crecimiento abdominal en una mujer en época reproductiva sugiere la posibilidad de embarazo; es así como el útero sometido a la acción mecánica del feto en crecimiento y a la acción hormonal, sobre todo de los estrógenos, experimenta una serie de cambios morfológicos que se manifiestan a partir de la semana 6-7 y es posible detectarlo mediante tacto vaginal. El útero aumenta de tamaño, sobre todo a expensas de su diámetro antero-posterior, y constituye el signo más confiable para el diagnóstico de embarazo durante el examen físico. ${ }^{5}$

A partir de la semana 12 el útero deja de ser un órgano pélvico y pasa a la cavidad abdominal, donde es posible su palpación por encima de la sínfisis púbica. Otro de los signos probables son los cambios cervicales, donde el cuello uterino es sometido a la acción hormonal y cambia de consistencia y color; así, a partir de la semana 5-6 se puede notar un reblandecimiento conocido como signo de Goodell. El cambio más notable, consiste en la aparición de una coloración violácea que adquiere el epitelio cérvicovaginal conocido como el signo de Jacquemier-Chadwick, que es debido a la congestión vascular y el signo de Hegar que consiste en el ablandamiento del istmo uterino. ${ }^{5}$

Por otra parte, en cuanto a las contracciones uterinas, existen dos tipos las descritas por Álvarez y Caldeyro, de poca intensidad, indoloras, con una frecuencia aproximada de una cada minuto y que sólo pueden ser detectadas por medio de equipos especiales; y las de Braxton-Hicks, también indoloras, de intensidad mayor que las anteriores, de frecuencia variable, generalmente una cada 10 minutos, y son las que producen la formación del segmento, la maduración del cuello y el descenso de la presentación. ${ }^{5}$ 
Por ultimo tenemos la palpación fetal mediante las maniobras de Leopold, las cuales permiten, en ausencia de equipos ultrasonográficos y de rayos $\mathrm{X}$, determinar la posición del feto. A su vez existen signos presuntivos de embarazo tales como amenorrea, nauseas, vómitos, fatiga, congestión de las mamas sobre todo en la primigrávida, asociado a la elevada producción de prolactina y cambios cutáneos como hiperpigmentación de los pómulos, conocida con el nombre de cloasma gravídico. ${ }^{5}$

Por lo antes descrito, la Organización Mundial de la Salud (OMS) concibe un mundo en el que todas las embarazadas y recién nacidos reciben una atención de calidad durante el embarazo, el parto y el periodo postnatal. En el continuo de servicios de atención de la salud reproductiva, la atención prenatal representa una plataforma para llevar a cabo importantes funciones de atención de la salud, como la promoción de la salud, el cribado y el diagnóstico, y la prevención de enfermedades. ${ }^{6}$

Así mismo, la UNICEF a nivel mundial, estima que el $86 \%$ de las mujeres embarazadas acceden a la atención prenatal con personal de salud capacitado al menos una vez, solo tres de cada cinco (62\%) reciben al menos cuatro visitas prenatales. En las regiones con las tasas más altas de mortalidad materna, como África subsahariana y Asia meridional, incluso menos mujeres recibieron al menos cuatro visitas prenatales (el $52 \%$ y el $46 \%$, respectivamente). ${ }^{7}$

Más aún, el Departamento de Obstetricia y Ginecología del Hospital Universitario de Chile adscrito a SEGO 2005 define al control prenatal como el conjunto de acciones y procedimientos sistemáticos y periódicos, destinados a la prevención, diagnóstico y tratamiento de los factores que pueden condicionar morbilidad materna y perinatal. Para obtener impactos sobre éstos, se requiere de un programa de alta eficacia, eficiencia, cobertura amplia y un manejo de partos con atención profesional sobre el $90 \%$. $^{8}$

Se debe agregar también, que el objetivo del control prenatal es lograr un embarazo que se desarrolle en los márgenes de normalidad física, psíquica, familiar y social culminando con un recién nacido y su madre sana. Los objetivos específicos son identificar factores de riesgo diagnosticar la edad gestacional, condición fetal y materna y educar a la madre. $^{8}$

Es así como RIGOL en su apartado de Obstetricia y Ginecología Cuba concuerda con los objetivos planteados por la OMS y SEGO, con la particularidad que incluye su 
propósito el cual consiste en lograr una óptima atención en salud para todas las gestantes, y que permita obtener un recién nacido vivo, sano, de buen peso y sin complicaciones maternas. ${ }^{9}$

Al mismo tiempo, en Venezuela la Norma Oficial para la atención integral de la Salud Sexual y Reproductiva define la Atención prenatal: Como un componente de la atención en Salud Sexual y Reproductiva que comprende un conjunto de acciones orientadas a lograr que el embarazo transcurra con vigilancia y asistencia por parte del equipo de salud según el riesgo identificado, a fin de controlar la evolución del embarazo y de preservar la salud de madre, hijo o hija durante su gestación. Toda mujer debe ser atendida indistintamente de la vestimenta que lleve, que tenga o no un documento de identidad, de la certeza de su embarazo, o de que haya perdido la cita. ${ }^{10}$

Cabe destacar que entre las principales características del control prenatal se encuentran: 1) PRECOZ: El primer contacto debe realizarse temprano, durante el primer trimestre de la gestación (en lo posible), ello permite la identificación del riesgo y ejecución oportuna de acciones de salud, la norma establece que para cumplir con este criterio el control de realizarse antes de la semana 13 mientras que un control tardío es aquel que se presenta luego de la semana 14. ${ }^{10}$

Consideraremos ahora: 2) PERIODICO: la frecuencia del control varía según el riesgo que presenta la embarazada siendo así para las embarazadas de Bajo Riesgo: Seis (6) consultas como mínimo, mientras que en las Alto Riesgo el número de Consultas dependerá de acuerdo al riesgo de la usuaria. El esquema de seis (6) Consultas, será de la siguiente manera: 1ra. Consulta: en el transcurso de las primeras 13 semanas, 2 da. Consulta: entre las 15 - 18 semanas, 3ra. Consulta: entre las 22 - 24 semanas, 4ta. Consulta: entre las 27 - 29 semanas, 5ta. Consulta: entre las 33 - 35, semanas 6ta. Consulta: entre las 38 - 40 semanas. Después de las 38 semanas de gestación se requiere efectuar controles prenatales semanales con el objeto de detectar, prevenir y tratar factores que aumenten el riesgo materno perinatal. ${ }^{10}$ Además, 3) COMPLETO: Los contenidos mínimos del Control Prenatal deberán garantizar el cumplimiento efectivo de las acciones de fomento, protección, recuperación y rehabilitación de la salud. 4) AMPLIA COBERTURA: Mientras mayor sea el porcentaje de embarazadas controladas, mayor será el impacto positivo sobre la morbilidad y mortalidad materna y perinatal. ${ }^{10}$ 
En cuanto a la clasificación del riesgo se hace en base a los siguientes factores: Edad menor de 18 años y mayor de 35 años, Paridad IV gestaciones o más, Intervalo intergenésico menor de 24 meses entre uno y otro embarazo, Patologías del embarazo, antecedente personales y familiares o presencia de alguna patología que determine riesgo, condiciones socioeconómicas, analfabetismo materno, pobreza crítica, unión inestable, entre otros. La presencia de uno o más de estos factores determinan el riesgo, permitiendo clasificarlo en 2 tipos alto y bajo riesgo obstétrico. ${ }^{10}$

Es así como las embarazadas de Bajo Riesgo son aquellas que evaluadas integralmente, presentan los criterios de condiciones óptimas para el bienestar de la madre y el feto y no se evidencia en ellas factores epidemiológicos de riesgo, antecedentes de patología ginecobstetricia perinatal o general, patología intercurrente, pérdidas reproductivas o embarazo no deseado, mientras que las embarazadas de alto riesgos son aquellas que presentan uno o más de los criterios o factores de riesgo antes nombrados y que ponen en peligro la vida de la madre y el feto. ${ }^{10}$

El alto riesgo obstétrico se puede dividir a su vez en tres tipos: Embarazo de Alto Riesgo Tipo I: se encuentran en buenas condiciones de salud, pero presentan uno o más factores de riesgo de tipo epidemiológico y/o sociales: Analfabetismo, Pobreza crítica, Vivienda no accesible al establecimiento de salud, Unión inestable, Trabajo con esfuerzo físico, Stress, Tabaquismo, alcohol, drogas, Embarazo no deseado, Edad Materna menor de 18 y mayor de 35 años, Talla menor de 150 centímetros, Peso materno menor de $45 \mathrm{Kg} \mathrm{u}$ obesidad. ${ }^{10}$

El embarazo de Alto Riesgo tipo II: Se encuentran en buenas condiciones de salud, pero presentan uno o más antecedentes de patología ginecobstétrica, perinatal o general, con o sin morbilidad materna y/o perinatal: Bajo peso al nacer, Peso inadecuado para la edad gestacional, Fetos Macrosómico, Malformaciones congénitas fetales, Parálisis cerebral, Madre Rh (-) no sensibilizada, Edad gestacional desconocida, Embarazo Cronológicamente prolongado, Preeclampsia o eclampsia, Cesárea anterior, Ruptura prematura de membranas, Distocias dinámicas, Hemorragias obstétricas o Mola hidatiforme. ${ }^{10}$

Dentro del embarazo de alto riesgo Tipo III, son las embarazadas de mayor riesgo y las que ameritan la atención más especializada. Se dividen en dos subtipos: IIIA) La 
gestación ocurre con patología gineco-osbtétrica, fetal o patología general, que afecta la salud materno-fetal sin antecedentes de morbilidad materna o mortalidad perinatal. ${ }^{10}$ Mientras el subtipo IIIB) Si presenta antecedentes de mortalidad perinatal como: Muertes perinatales, Síndrome de TORCHS (Citomegalovirus, Rubeola, Toxoplasmosis, Herpes simple tipo I y II, Sífilis), Salmonellosis, Gonorrea, Candidiasis, estreptococos A y B, Tuberculosis, Chagas, Paludismo, VIH/SIDA.) Isoinmunización, Abortadora habitual, Infertilidad Incompetencia cervical, Malformaciones congénitas uterinas, Tumores ginecológicos, Cáncer extragenital, Hipertensión arterial, Preeclampsia/eclampsia, Hemorragias obstétricas, Presentaciones anormales, Estrechez pélvica, Placenta previa, Amenaza de parto prematuro, Embarazo múltiple, Anemia, Cardiopatías, Nefropatías Diabetes, Colagenosis, hemoglobinopatías, Desnutrición severa o Psicopatías. ${ }^{10}$ Por lo antes expuesto, el Ministerio del Poder Popular para la Salud-MPPS en su Protocolo de atención, cuidados prenatales y atención obstétrica de emergencia en su Primera edición de Marzo 2014, establece que la primera consulta iniciara con la elaboración de una historia clínica detallada que contenga antecedentes familiares y personales de la paciente, haciendo hincapié en antecedentes ginecológicos: edad de la menarquía, tipo menstrual, inicio de las relaciones sexuales, número de parejas, infecciones de transmisión sexual, uso de métodos anticonceptivos, últimas citologías, así como obstétricos: curso de los embarazos, patologías presentadas, posibles complicaciones, peso y talla de hijos anteriores. ${ }^{11}$

De igual modo, se deberá realizar un examen físico integral, que abarque presión arterial, peso, talla, índice de masa corporal, examen buco dental, de tórax, mamas, abdomen, y extremidades, pero sobre todo con énfasis en la exploración obstétrica: altura uterina, movimientos, latidos cardiacos fetales y toma de citología. ${ }^{11}$

Entre los principales exámenes de laboratorio solicitados al inicio del control se encuentran: hematología completa, urea, creatinina, glicemia, VDRL, VIH, examen de heces, examen de orina, urocultivo, grupo sanguíneo, factor $\mathrm{Rh}$, antígeno de superficie de la hepatitis B, Serología para toxoplasmosis ( $\lg G$ e $\lg M$ ) y rubéola ( $\lg G$ ) (en caso de no haber sido vacunada). En caso de Rh negativo: tipiaje de la pareja, aglutininas anti Rh. Ecosonograma básico en base al cual se establecerá la edad gestacional, vitalidad 
embrionaria así como la localización del saco gestacional, número de fetos, cantidad de líquido amniótico y ubicación placentaria. ${ }^{11}$

En este sentido, se indica exploraciones especiales para descartar malformaciones congénitas del feto: Pesquisa ecográfica de aneuploidias: Translucencia nucal: Entre la semana 11 y 14, medición del pliegue nucal cerca de la semana 14 y 19, pesquisa bioquímica de aneuploidias: PAPP-A, B hCG, alfafetoproteína a la semana 11 y 14, estriol en la semana 14 y 19 y Amniocentesis para estudio genético entre la semana 16 y 19 en las pacientes mayores de 35 años. ${ }^{11}$

Mientras que en las consultas sucesivas se reinterrogará a la gestante, se practicará un examen clínico exhaustivo haciendo hincapié en el índice de masa corporal (IMC), de acuerdo con el siguiente esquema: Bajo peso (IMC menor de 19,8), Peso normal (IMC 19,9 - 24,8), Sobrepeso (IMC 24,9 - 29,9), Obesidad (IMC mayor a 30). Toma de Presión arterial, evaluando posibles de signos de preeclampsia, así como medición de la altura uterina, estimación de la estática fetal a partir Maniobras de Leopold y auscultación del latido cardíaco fetal. La exploración vaginal: se reserva para aquellos casos en los que la clínica sugiera su utilidad. ${ }^{11}$ Igualmente, el protocolo también especifica la reanudación de los exámenes antes nombrados en las semanas 24-28 de gestación hasta la semana 32-36. Todo esto con el fin de reducir en lo posible la tasa de morbimortalidad materno fetal y llegar a la culminación del parto sin ninguna clase de complicación. ${ }^{11}$

Al mismo tiempo, la (OMS) también establece una serie de recomendaciones en el contexto de la atención prenatal rutinaria, A.1.1 Intervenciones alimentarias: para brindar asesoramiento sobre la importancia de una alimentación saludable y la actividad física durante el embarazo. A.2.1 Uso de suplementos de hierro y ácido fólico: Para prevenir la anemia materna, la sepsis puerperal, el bajo peso al nacer y el nacimiento prematuro. A.3 Suplementos de calcio: En poblaciones con una dieta baja en calcio, se recomienda que las embarazadas tomen un suplemento diario de calcio $(1,5-2,0 \mathrm{~g}$ de calcio elemental por vía oral) para reducir el riesgo de preeclampsia. ${ }^{12}$

A.4 Suplementos de vitamina A: Solo se recomienda la toma de un suplemento de vitamina A como método para prevenir la ceguera a las embarazadas de zonas donde el déficit de vitamina A sea un problema grave de salud pública. B.1.1 hemograma completo: recomendado para diagnosticar la anemia. y B.1.2 cultivo de muestras de orina para 
diagnosticar la bacteriuria asintomática (BA) en embarazadas. En entornos donde no es posible realizar urocultivos, para diagnosticar la BA en embarazadas se recomienda realizar in situ la tinción de Gram. ${ }^{12}$

Teniendo en cuenta lo anteriormente expuesto, se han realizado una serie de estudios, dirigidos a profundizar acerca de los factores asociados a la no asistencia al control prenatal y causas de abandono del mismo. Primeramente el trabajo de investigación presentado por Salcedo, Yanet, (2017) Perú, titulado; "Factores sociodemográficos asociados a un inadecuado control prenatal en las puérperas del Hospital Sergio E. Bernales. 2015 - 2016", con 264 puérperas. Los resultados fueron: existen factores epidemiológicos que muestran tendencia asociada de tener riesgo de un inadecuado control prenatal como: rango de edad de 14 a 18 años $(\mathrm{p}=0.009)$, más de tres hijos $(p=0.72)$ y grado de instrucción primaria $(p=0.726) .{ }^{13}$

Entre de los factores socioeconómicos: la dependencia económica $(p=0.62)$ y vivienda zona rural $(p=0.45)$ muestran tendencia asociada a aumentar el riesgo, mientras que el grado de instrucción primaria reduce el riesgo de tener inadecuado control prenatal $(p=0.6)$ y dentro de los factores maternos el no tener co-morbilidad durante la gestación muestra tendencia a estar asociado a ser un factor protector de no tener un inadecuado control prenatal $(p=0.23) .{ }^{13}$

De igual manera, César García-Balaguera. (2016), Colombia, en un trabajo tiulado "Barreras de acceso y calidad en el control prenatal", con una muestra de 15.537 gestantes. Evaluaron las barreras de acceso local, donde, ingresar al centro de atención fue cercano y fácil en cuanto al transporte para el $40.13 \%$, lejos pero fácil para el $42.43 \%$ y lejos y difícil para el $17.43 \%$. Las instalaciones donde atendieron a las madres se calificaron como excelentes por el $8.28 \%$, buenas por el $84.11 \%$, regulares por el $6.29 \%$ y malas por el $1.32 \%$. Respecto al horario de atención, al $84.77 \%$ le pareció conveniente y al $15.3 \%$ no le convino. Un $3.64 \%$ refirió cobro por el CPN y 96.36 no tuvo cobro alguno (en el sistema de salud colombiano el CPN no tiene cobro). ${ }^{14}$

El tiempo de espera para la atención correspondió a 13.58\% inmediato, 52.65\% de 5 a 15 minutos, $25.17 \%$ de 15 a 30 minutos y $8.61 \%$ mayor a 30 minutos. El $88.31 \%$ tuvo CPN por parte de un médico y $11.75 \%$ por una enfermera o auxiliar de enfermería. Respecto al acceso a servicios complementarios, el $30.77 \%$ tuvo inconvenientes para la 
autorización o entrega de las ordenes de laboratorio clínico y el $69.23 \%$ no presentó problema alguno. Para la autorización o acceso al ginecólogo, el $37.18 \%$ tuvo dificultades y $62.82 \%$ no las tuvo. ${ }^{14}$

Así mismo, Flores, Sybil (2017) en Perú realizaron un estudio titulado "Nivel de conocimiento sobre la atención prenatal en mujeres de 20 a 40 años de edad", evaluando a 142 mujeres. Los resultados obtenidos fueron: el 55.6\% (79) tenían edades comprendidas entre los 20 a 29 años de edad; con nivel de instrucción secundaria $(60.6 \%)$; convivientes $(52.8 \%)$, el $19.7 \%$ se encontraban embarazadas, multíparas (33.1\%); y el 53.5\% con menos de 6 controles prenatales en su último embarazo. ${ }^{15}$

El $45.8 \%$ presentaron un nivel de conocimiento adecuado sobre la atención prenatal, el mejor nivel de conocimiento se asoció con la edad de 30 a 40 años de edad ( $p=0.005)$; con el nivel de instrucción técnica/superior ( $p=0.025)$; con las mujeres que se encuentran embarazadas ( $p=0.002)$; con la multíparidad $(p=0.004)$; y con las que tienen de 6 a más controles prenatales $(p=0.000)$. En conclusión menos del $50 \%$ de las mujeres entre 20 a 40 años del Asentamiento humano (AAHH) La Paz del distrito de Punchana presentan un adecuado conocimiento sobre el concepto y actividades de la atención prenatal. ${ }^{15} \mathrm{En}$ el ámbito nacional no se encontraron trabajos actuales publicados acerca de los factores relacionados al no cumplimiento del control prenatal.

Finalmente, Soler Y, Vilchez, D, Vilchez D. (2013) en Dabajuro, Edo Falcón cuyo objetivo fue "Determinar los Factores contributivos para el control prenatal tardío en mujeres con un rango de edad entre 14 y 43 años". Presentaron un estudio de tipo descriptivo, de campo de corte transversal, incluidas 186 pacientes obstétricas, pertenecientes al registro de control prenatal, Hospital tipo I "Dr. José Enrique Zavala" de Dabajuro, Edo Falcón. Entre los principales resultados fueron: Prevalencia 44.44\%, control tardío e irregular; unión libre: $31.8 \%$; adolecentes 29.9\%; embarazo no planificado $67 \%$; inició el CPN; entre el II-III trimestre el 15.8\%. ${ }^{16}$

Nivel de instrucción 39.3\%; diversificado y amas de casa 45.7\%; Si conocían sobre el control prenatal $79.7 \%$; la importancia del control prenatal, recae en que evita complicaciones manifestó $45.7 \%$; intervalo de consultas Bimensual $22.3 \%$; el $63.63 \%$ refirió haber sido víctima de algún tipo de violencia de las cuales $78 \%$ refirió violencia psicológica, verbal y familiar. Demora en la 1ra consulta 57.4; Conclusión: El poco 
ingreso económico, el nivel de instrucción y bajo conocimiento sobre el control prenatal y su importancia interfieren en el inicio oportuno de las consultas prenatales. ${ }^{16}$

En definitiva, la atención prenatal reduce la morbilidad y mortalidad materna y perinatal, partos prematuros y el número de productos con bajo peso al nacer; también permite identificar factores de riesgo, lo cual hace posible establecer acciones preventivas terapéuticas oportunas durante el embarazo, lo que se traduce en un menor costo en su atención posterior y mejoras en la calidad de vida de las gestantes y sus hijos. Así que, la adherencia de la embarazada al control prenatal comprende tanto la asistencia al control, como el cumplimiento de las recomendaciones dadas. ${ }^{17,18}$

Por lo anteriormente expuesto surge la inquietud de conocer que factores que influyen en la no adherencia al control prenatal en gestantes adscritas al Ambulatorio Manuel Navas Lizardo de Capatárida Municipio Buchivacoa, Estado Falcón, durante el lapso académico Agosto 2018 - Enero 2019 y se formula la siguiente interrogante: ¿Cuál son los factores asociados a la no adherencia al control prenatal en gestantes adscritas al ambulatorio Manuel Navas Lizardo, Capatárida Municipio Buchivacoa?

\section{Objetivo general}

Determinar cuáles son los factores asociados a la no adherencia al control prenatal en gestantes adscritas al ambulatorio Manuel Navas Lizardo, Capatárida Municipio Buchivacoa en el lapso Agosto 2018 - Enero 2019.

\section{Objetivos específicos}

Identificar las características sociodemográficas de las gestantes adscritas al programa de control prenatal del ambulatorio Manuel Navas Lizardo.

Establecer las limitaciones que tienen las gestantes para asistir al control prenatal.

Evaluar el nivel de conocimiento que tienen las gestantes sobre el control prenatal.

Determinar cuáles son los factores asociados a la no adherencia al control prenatal en gestantes adscritas al ambulatorio Manuel Navas Lizardo, Capatárida Municipio Buchivacoa en el lapso Agosto 2018 - Enero 2019. 


\section{METODOLOGIA}

El tipo de estudio es descriptivo, de corte transversal y de campo, con un diseño no experimental.

La población estuvo conformada por todas las embarazadas de la Parroquia Capatárida, Municipio Buchivacoa, Estado Falcón dando un total de 50 mujeres. La muestra fue intencional no probabilística que cumplieran con los siguientes criterios de inclusión.

\section{Criterios de inclusión:}

Gestantes que habiten en la Parroquia Capatárida, Municipio Buchivacoa, Estado Falcón. Gestantes que estuvieran dispuestas a formar parte de la investigación y a firmar el consentimiento informado.

\section{Criterios de exclusión:}

Gestantes que no habiten en la Parroquia Capatárida, Municipio Buchivacoa, Estado Falcón.

Mujeres no embarazadas

\section{Técnica e Instrumento de recolección de datos}

El presente trabajo de investigación, inicio con la entrega de una solicitud de permiso al coordinador del ARII "Manuel Navas Lizardo" de la Parroquia Capatárida Municipio Buchivacoa, Estado Falcón donde se le informo la realización de dicho estudio. (Anexo 1).

Una vez identificada la paciente embarazada fue inicialmente catalogada bajo los criterios de inclusión y exclusión, y posteriormente se le dio a conocer los aspectos de la investigación explicándoles el motivo del trabajo e invitándolas a participar de tal forma de obtener su autorización a través de un consentimiento informado (Anexo 2) el cual expuso de forma precisa los aspectos del proyecto.

Luego de obtener las respectivas autorizaciones, se procedió a la recolección de la información a través de una ficha de recolección de datos con el fin de determinar los factores sociodemográficos, por medio de la escala Graffar modificada de Méndez Castellano (Anexo 3). La cual consto de 4 variables a utilizar para la obtención de la 
estratificación social: Profesión del jefe del hogar, nivel de instrucción de la esposa, principal fuente de ingresos del hogar y condiciones de alojamiento, la suma del valor obtenido de las 4 variables determino el Estrato del hogar según la escala.

A continuación se aplicó un instrumento para la recolección de datos, modificado según requerimientos de este estudio, el cual se extrajo de los trabajos de investigación "Factores asociados a la no adherencia al programa de control prenatal en las gestantes captadas de la Empresa Social del Estado Prestadora de Servicios de Salud (ESE) Hospital Sabana de Torres" Realizado por Alix Lozano, Alba Cala, Heidy Molina. (2011) y "Factores asociados a la inasistencia al control prenatal durante el primer trimestre en mujeres gestantes de la (ESE) San Sebastián La Plata Huila" Realizado por Lina Escobar, Lyda Guzmán, y Ramiro Córdoba. (2009) los cuales fueron previamente validados (Anexo 4) ${ }^{19,20}$. Este instrumento fue diseñado en dos partes; la primera conformada por: Datos personales, estado civil, área de procedencia, nivel de educación, y antecedentes gineco-obstétricos, que permitieron obtener más datos sobre las características sociodemográficas de las gestantes; La segunda parte, por una serie de preguntas de selección simple, en las cuales la gestante según su situación, seleccionaron cuales son las limitaciones o barreras que tienen para acceder al control prenatal. Finalmente para evaluar el nivel de conocimiento que tienen las gestantes adscritas al control prenatal del ambulatorio Manuel Navas Lizardo se aplicó a la muestra de participantes, un instrumento de medición (prueba de conocimiento), creado por los autores previamente validado por un Metodólogo Estadista y un Médico Gineco-obstetra. Constituido por 10 preguntas de selección única, referente a los objetivos, características, tipo, inicio, y exámenes a realizar en el control prenatal. Cada pregunta consto de varias opciones, siendo solo una de ellas la respuesta correcta, a la cual se le atribuyo el valor de un 1 punto, produciendo una escala de 0 a 10 puntos, lo que permitió categorizar el nivel de conocimiento de las gestantes acerca del control prenatal en Deficiente: de 0 a 3 puntos, Regular: de 4 a 7 puntos y Alto: de 8 a 10 puntos.

\section{Técnica de Análisis de los Datos}

Para el análisis de los datos recolectados posterior a la aplicación de los correspondientes instrumentos se utilizaron cuadros, que fueron organizados y tabulados para su análisis 
a través del Programa Estadístico Computarizado: International Business Machines Statistical Package for the Social Sciences (IBM -SPSS) versión 21, en donde se calcularan las distribuciones de frecuencia y porcentajes para las variables de estudio; Así como los estadios descriptivos correspondientes a cada uno de los métodos utilizados para determinar los factores relacionados a la no adherencia al control prenatal en las gestantes de la Parroquia Capatárida, Municipio Buchivacoa, Estado Falcón.

\section{RESULTADOS}

\section{CUADRO № 1}

DISTRIBUCION DE LA MUESTRA DE ACUERDO LAS CARACTERISTICAS SOCIODEMOGRAFICAS

\begin{tabular}{|c|c|c|c|}
\hline $\begin{array}{l}\text { CARACTERISTICAS } \\
\text { SOCIODEMOGRAFICAS }\end{array}$ & $\begin{array}{l}\text { ALTERNATIVAS } \\
\text { (DETALLES) }\end{array}$ & FRECUENCIA & PORCENTAJE \\
\hline \multirow{4}{*}{$\begin{array}{c}\text { EDAD } \\
(23,52 \pm 7,98)\end{array}$} & MENOR DE 20 AÑOS & 22 & $44 \%$ \\
\hline & DE 20 A 29 AÑOS & 19 & $38 \%$ \\
\hline & DE 30 A 39 AÑOS & 7 & $14 \%$ \\
\hline & MAYOR DE 39AÑOS & 2 & $4 \%$ \\
\hline \multirow{4}{*}{ ESTADO CIVIL } & SOLTERA & 17 & $34 \%$ \\
\hline & CASADA & 10 & $20 \%$ \\
\hline & DIVORCIADA & 1 & $2 \%$ \\
\hline & UNION LIBRE & 22 & $44 \%$ \\
\hline \multirow{4}{*}{ NIVEL DE INSTRUCCIÓN } & PRIMARIA & 10 & $20 \%$ \\
\hline & SECUNDARIA & 25 & $50 \%$ \\
\hline & TECNICA & 2 & $4 \%$ \\
\hline & UNIVERSITARIA & 13 & $26 \%$ \\
\hline \multirow{2}{*}{ PROCEDENCIA } & RURAL & 0 & $0 \%$ \\
\hline & URBANA & 50 & $100 \%$ \\
\hline \multirow{3}{*}{ NIVEL SOCIOECONOMICO } & ESTRATO I & 2 & $4 \%$ \\
\hline & ESTRATO II & 5 & $10 \%$ \\
\hline & ESTRATO III & 16 & $32 \%$ \\
\hline
\end{tabular}




\begin{tabular}{|c|c|c|}
\hline ESTRATO IV & 13 & $26 \%$ \\
\hline ESTRATO V & 14 & $28 \%$ \\
\hline
\end{tabular}

FUENTE: FICHA DE RECOLECCION DE DATOS

CUADRO № 2

DISTRIBUCION DE LA MUESTRA DE ACUERDO LOS ANTECEDENTES GINECO-OBSTETRICOS DE LAS GESTANTES

\begin{tabular}{|c|c|c|c|}
\hline VARIABLES & DETALLES & FRECUENCIA & PORCENTAJE \\
\hline \multirow{5}{*}{$\begin{array}{l}\text { SEMANAS DE } \\
\text { GESTACION }\end{array}$} & $\leq 12$ SEMANAS & 1 & $2 \%$ \\
\hline & 13 A 20 SEMANAS & 3 & $6 \%$ \\
\hline & 21 A 27 SEMANAS & 10 & $20 \%$ \\
\hline & 28 A 32 SEMANAS & 10 & $20 \%$ \\
\hline & 33 A 40 SEMANAS & 26 & $52 \%$ \\
\hline \multirow{2}{*}{ INICIO CONTROL } & PRECOZ & 1 & $2 \%$ \\
\hline & TARDIO & 49 & $98 \%$ \\
\hline \multirow{3}{*}{$\begin{array}{l}\text { NUMERO DE } \\
\text { CONTROLES }\end{array}$} & $\leq 4$ CONTROLES & 42 & $84 \%$ \\
\hline & 5 A 7 CONTROLES & 2 & $16 \%$ \\
\hline & $\geq 8$ CONTROLES & 0 & $0 \%$ \\
\hline \multirow{3}{*}{$\begin{array}{l}\text { NUEMERO DE } \\
\text { GESTAS }\end{array}$} & I GESTA & 30 & $60 \%$ \\
\hline & II - III GESTAS & 15 & $30 \%$ \\
\hline & $\geq 4$ GESTAS & 5 & $10 \%$ \\
\hline
\end{tabular}

FUENTE: FICHA DE RECOLECCION DE DATOS 
CUADRO № 3

DISTRIBUCION DE LA MUESTRA DE ACUERDO A LAS LIMITACIONES QUE TIENEN LAS GESTANTES PARA ASISTIR AL CONTROL PRENATAL

\begin{tabular}{|c|c|c|c|}
\hline VARIABLES & DETALLES & FRECUENCIA & PORCENTAJE \\
\hline \multirow{7}{*}{$\begin{array}{l}\text { ¿Por cuales motivos } \\
\text { usted dejo de asistir } \\
\text { a alguna de las citas } \\
\text { del control prenatal? }\end{array}$} & MALA ATENCION & 2 & $4 \%$ \\
\hline & FALTA DE DINERO & 10 & $20 \%$ \\
\hline & TRANSPORTE & 10 & $20 \%$ \\
\hline & AMBULATORIO LEJOS & 10 & $20 \%$ \\
\hline & POCO IMPORTANTE & 5 & $10 \%$ \\
\hline & VA A OTRO LUGAR & 3 & $6 \%$ \\
\hline & $\begin{array}{c}\text { NO HA DEJADO DE } \\
\text { ASISTIR }\end{array}$ & 10 & $20 \%$ \\
\hline \multirow{5}{*}{$\begin{array}{c}\text { ¿Qué dificultades ha } \\
\text { tenido para obtener } \\
\text { una cita al control } \\
\text { prenatal? }\end{array}$} & FILA DE ESPERA & 22 & $44 \%$ \\
\hline & DOCUMENTOS CITA & 21 & $42 \%$ \\
\hline & ERROR ASIGNACION & 3 & $6 \%$ \\
\hline & $\begin{array}{c}\text { AUSENCIA } \\
\text { PROFESIONAL }\end{array}$ & 3 & $6 \%$ \\
\hline & NINGUNA & 1 & $2 \%$ \\
\hline \multirow{5}{*}{$\begin{array}{l}\text { ¿Cuándo usted } \\
\text { solicito la cita para } \\
\text { asistir al control } \\
\text { prenatal se la } \\
\text { asignaron? }\end{array}$} & DE INMEDIATO & 5 & $10 \%$ \\
\hline & 1-3 DIAS & 20 & $40 \%$ \\
\hline & 4 - 10 DIAS & 15 & $30 \%$ \\
\hline & 11 - 30 DIAS & 8 & $16 \%$ \\
\hline & MAS DE 1 MES & 2 & $4 \%$ \\
\hline
\end{tabular}

FUENTE: FICHA DE RECOLECCION DE DATOS 
CUADRO № 4

DISTRIBUCION DE LA MUESTRA DE ACUERDO AL NIVEL DE INFORMACION SOBRE DEL CONTROL PRENATAL

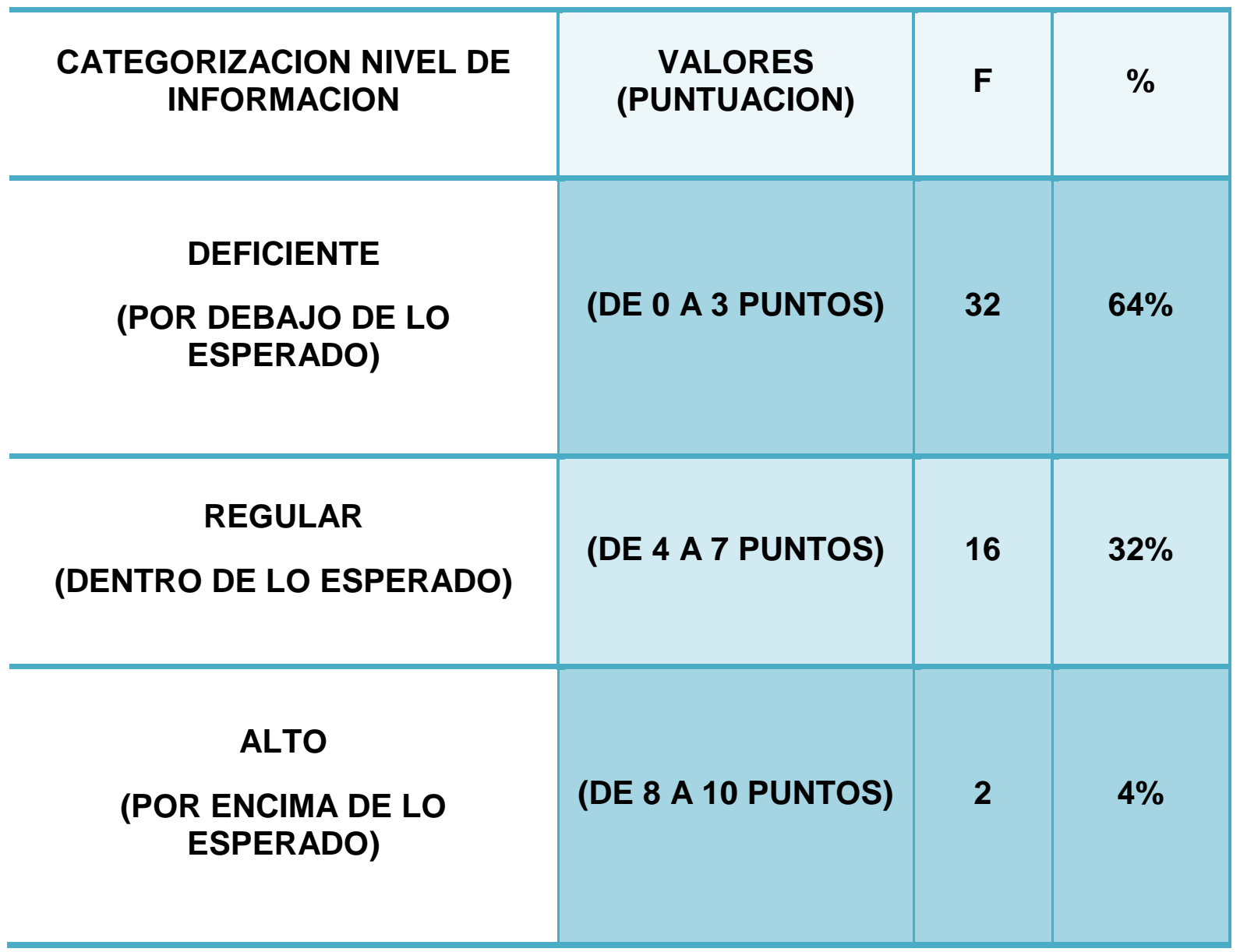

FUENTE: FICHA DE RECOLECCION DE DATOS

\section{ANÁLISIS}

En cuanto a las características sociodemográficas se encontró que la muestra de mujeres que participaron en este estudio tenía una edad promedio de 23,52 años, con una desviación estándar de \pm 7,98 años, para una edad mínima de 14 y máxima de 42, el grupo etario fue: menor de 20 años con (44\%) seguido 20 - 29 (19\%), de 30-39 (7\%), y mayor 39 (2\%). Cuyo grado de instrucción demostró: (50\%) secundaria, (20\%) primaria; (26\%) universitaria y solo el (4\%) Técnica superior, con respecto al estado civil: (44\%) viven en unión libre, (34\%) son solteras, el (20\%) casada; y (2\%) divorciada. Al obtener 
las características socioeconómicas predomino el estrato III con (32\%) seguido por estrato V pobreza crítica (28\%) y estrato IV pobreza relativa (26\%). (Tabla № 1 )

De acuerdo a las semanas de gestación en que se encontraban las embarazadas al momento de este estudio: (52\%) entre la $33-40$, (20\%) de la $28-32$, otro (20\%) $21-27$, (6\%) $13-20$ y (2\%) menor a 12 semanas. Al evaluar el inicio al control prenatal: (98\%) fue tardío, mientras el (2\%) precoz. En cuanto al número de controles el (84\%) presento menos de 4 controles, (16\%) de 5- 7 controles y $(0 \%)$ más de 8 controles. Según el número de gestas: (60\%) fueron primigestas, el (30\%) se encontraba entre su II- III gesta y solo el (10\%) más de IV gestas. (Tabla № 2)

Con relación a las limitaciones que tienen las gestantes para asistir al control prenatal, destacan: falta de dinero, dificultad para conseguir transporte, distancia del ambulatorio (señalado por el $20 \%$ ), al (10\%); no le parece importante, (6\%) le gusta la atención en otro lugar, (4\%) arrojo mala atención por parte del personal y el $(20 \%)$ restante, no señalaron ningún motivo o factor para dejar de asistir. Entre las dificultades para conseguir la cita se evidencio: filas de espera (44\%), documentos necesarios para solicitud de la cita (42\%); Error en asignación de cita (6\%); ausencia de profesional (6\%) ninguna causa aparente (2\%). Así mismo el tiempo estimado entre la solicitud de la cita y la realización del control prenatal, oscilo de 1 a 3 días en el (40\%), de 4 a 10 días (30\%) de 11 a 30 días (16\%), un (10\%) de las gestantes afirmo que el control fue de inmediato a la solicitud y solo (4\%) indicaron una espera de más de 1 mes. (Tabla № 3 ) Al medir el nivel de conocimiento de las participantes acerca del control prenatal, se obtuvo un promedio de 3,54 puntos, con una desviación estándar de $\pm 1,66$ puntos, con un puntaje mínimo de 1 y un máximo de 9, para una amplitud de 8 puntos. Analizando cada una de las puntuaciones obtenidas por las participantes se pudo determinar que: el $64 \%$ poseen un nivel de conocimiento deficiente, $32 \%$ un nivel de conocimiento regular, y solo el $4 \%$ mostro un nivel de conocimiento alto. (Tabla № 4)

\section{DISCUSIÓN DE LOS RESULTADOS}

El grupo etario predominante fue la edad comprendida menor a 20 años (44\%), seguido 20-29 (38\%), de 30-39 años (14\%), y mayor de 39 años (4\%). Datos concordantes con Salcedo, Yanet, (2017) en donde el rango de edad fue 14 a 18 años $(p=0.009)^{13}$. Pero 
que no se relacionan con Flores, Sybil (2017) donde el grupo etario se ubicó entre 20- 29 años de edad. ${ }^{15}$ El estado civil, fue unión libre con (44\%) lo cual guarda relación con los estudios de Soler Y, Vilchez, D, Vilchez D. (2013) y Flores, Sybil (2017) con (31.8\%) y (52.8\%). ${ }^{16,15}$ Con relación al nivel de instrucción (50\%) fue educación secundaria, datos concordantes con Flores, Sybil (2017) en un (60.6\%) ${ }^{15}$ al contrario de Salcedo, Yanet, (2017) donde el grado de instrucción fue primaria $(p=0.726) \cdot{ }^{13}$ En cuanto al nivel socioeconómico el grupo etario predominante se ubicó en (Estrato III) con (32\%) seguido por (Estrato V) pobreza crítica con (28\%) y (Estrato IV) pobreza relativa (26\%.) Sin embargo se constató que no existen investigaciones previas que permitan la discusión de este hallazgo. Al evaluar el inicio del control prenatal el (98\%) fue tardío, del mismo modo Soler Y, Vilchez, D, Vilchez D. (2013) detectaron un inició tardío; entre el II-III trimestre con 15.8\%. ${ }^{16}$. Según el número de controles: (84\%) presento menos de 4, datos acordes al trabajo realizado por Flores, Sybil (2017) en donde el (53.5\%). presento menos de 6 controles. ${ }^{15}$

El número de gestas fue: (60\%) primigestas, (30\%) se encontraba entre su II- III gesta y solo el (10\%) tenían más de IV gestas. Datos discordantes con Flores, Sybil (2017) donde $(33.1 \%)$ eran multíparas. ${ }^{15}$ En referencia a las limitaciones que tienen las gestantes para asistir al control prenatal, están: falta de dinero, dificultad para conseguir transporte, y distancia al ambulatorio con (20\%). Lo que contrasta con el estudio de César García-Balaguera. (2016) en donde ingresar al centro de atención fue cercano y fácil para el $(40.13 \%)$ de las gestantes. ${ }^{14}$

Entre las dificultades, señaladas para conseguir la cita al control destacan: filas o colas de espera (44\%) y documentos necesarios para solicitud de la cita (42\%). Lo que difiere con el tiempo de atención arrojado por César García-Balaguera (2016). Obteniendo atención inmediata en el (13.58\%) de los casos, y de 5 a 15 minutos en el (52.65\%). ${ }^{14}$ Finalmente el nivel de conocimiento de las gestantes acerca del control prenatal, se pudo determinar que el $64 \%$ poseen un nivel de conocimiento bajo. Datos que difieren del estudio realizado por Flores, Sybil (2017) donde (45.8\%) presentaron un nivel de conocimiento adecuado. ${ }^{15}$ 


\section{CONCLUSIONES}

En cuanto a las características sociodemográficas de las gestantes que acuden al ambulatorio Manuel Navas Lizardo, se encontró edad promedio de 23,52 años, con una desviación estándar de $\pm 7,98$ años, para una edad mínima de 14 y máxima de 42 . El grupo etario predominante fue menor de 20 años, seguido de $20-29,30-39$ y por ultimo mayor a 39. Con nivel de instrucción secundaria, estado civil unión libre, estrato socioeconómico III, procedencia rural, inicio tardío al control, menos de 4 consultas y primigestas. Las principales limitaciones para acceder al control prenatal fueron: factor económico, transporte, distancia al centro de salud, filas de espera, falta de documentación solicitada, tiempo de espera entre la solicitud de la cita y la realización del control y ausencia de personal.

El nivel de conocimiento de las gestantes sobre el control prenatal arrojo ser deficiente, debido a falta de información, lo que puede conllevar a la aparición de complicaciones materno-fetales, por lo que es necesario promover diálogos, charlas o talleras por parte del personal de salud acerca de la importancia de asistir a consulta prenatal, con el fin de orientar, y brindar una atención oportuna.

\section{RECOMENDACIONES}

Incentivar a las gestante por medio de diálogos interactivos, conversatorios y talleras acerca de la importancia de asistir a consulta de control prenatal.

Promover en el personal de salud estrategias que permitan agilizar la documentación y asignación oportuna de las citas.

Realizar visitas domiciliarias a embarazadas de escasos recursos y domicilio lejano que no puedan acceder al control prenatal.

Crear un club de embarazadas, donde se les brinde atención, información, y recreación con fin de captar nuevas gestantes a la consulta. 


\section{REFERENCIAS CONSULTADAS}

1. Atrash HK, Friede A, Hogue CJR. «Abdominal Pregnancy in the United States: Frequency and Mortality». Disponible https://es.wikipedia.org/wiki/Embarazo humano\#cite ref-Atrash 5-0.

Consultado 5/07/2018

2. Comunicado del Comité de Bioética de la Comisión de Familia de la Conferencia Episcopal Peruana. Disponible https://es.wikipedia.org/wiki/Embarazo humano\#cite ref-9. Consultado $5 / 07 / 2018$

3. Menéndez, G. G. E., Navas, C. I., Hidalgo. El embarazo y sus complicaciones en la madre adolescente. Disponible https://es.wikipedia.org/wiki/Embarazo humano\#cite ref-4. Consultado $5 / 07 / 2018$

4. Gabbe SG, Niebyl JR, Simpson JL. Obstetrics - Normal and Problem Pregnancies (5 $\quad$ edición). Disponible https://es.wikipedia.org/wiki/Embarazo humano\#cite ref-32. Consultado $5 / 07 / 2018$

5. Juan Aller Gustavo Pagés. Diagnóstico De Embarazo. En: Juan Aller. Editor. Obstetricia Moderna. Ed. McGraw-Hill-Interamericana. 3edicion Pag 2 -10.

6. Organización mundial de la Salud "Recomendaciones de la OMS sobre atención prenatal para una experiencia positiva del embarazo" Ed 2016 Pag 1

7. Fondo de las Naciones Unidas para la infancia. Disponible https://data.unicef.org/topic/maternal-health/antenatal-care/\#. Consultado 5/07/2018

8. Guido Juarez, Carlas Jadue. Control prenatal. En: Hugo Salina, Mauro Parra, Enrique Valdes. Editores. Obstetricia. Departamento de Obstetricia y Ginecología Hospital Clínico Universidad de Chile Ed. 2005 Pag 5

9. S. Santisteban. Atención Prenatal. En: Orlando Rigol Ricardo. Editor. Obstetricia y Ginecologia. Ed. La Habana: Editorial Ciencias Médicas; 2004. Pag79

10. República Bolivariana de Venezuela, Ministerio de Salud y Desarrollo Social, "Norma Oficial Atención prenatal Para La Atención integral de la Salud Sexual y Reproductiva" Tomo III: Manual de procedimientos, Atención prenatal. Caracas: MSDS; 2004. 
11. República Bolivariana de Venezuela, Ministerio del Poder Popular para la Salud-MPPS. Protocolos de atención. Cuidados prenatales y atención obstétrica de emergencia. Caracas: MPPS; 1ra edición: Marzo 2014.

12. Organización mundial de la Salud "Recomendaciones de la OMS sobre atención prenatal para una experiencia positiva del embarazo" Ed 2016. Pag 3-4

13. Salcedo Campos, Yanet Elvira. Factores sociodemográficos asociados a un inadecuado control prenatal en las puérperas del Hospital Sergio E Bernales en el periodo de mayo del 2015 a mayo del 2016. [Tesis Doctoral] Lima- Perú. Ed 2017.

14. César García-Balaguera. Barreras de acceso y calidad en el control prenatal. Universidad Cooperativa de Colombia - Sede Villavicencio - Facultad de Medicina - Villavicencio - [Tesis Doctoral] Colombia. Ed 2016.

15. Sybil Carolina Flores Torres. Nivel de conocimiento sobre la atención prenatal en mujeres de 20 a 40 años de edad del AAHH la Paz-Punchana Marzo 2016. [Tesis Doctoral] San Juan- lquitos-Perú. Ed 2017.

16. Soler Ynes, Vilchez Daycelyn, Vilchez Daysiree. Factores contributivos para el control prenatal tardío en mujeres con un rango de edad entre 14 y 43 años. Hospital tipo I "D.r José Enrique Zavala" de Dabajuro. [Tesis Doctoral]. Venezuela Edo Falcón. Ed 2013.

17. Sánchez-Nuncio Héctor, Pérez Toga Gustavo, Pérez Rodríguez Francisco. Impacto del control prenatal en morbilidad y mortalidad neonatal. Revista Médica del IMSS. Vol 43, N 5. Sep-Oct2005.

18. Flor de María Cáceres-Manrique, Articulo de reflexión: El control Prenatal: Reflexión urgente. Prenatal care: an urgent reflection, MSc. Revista Colombiana de Obstetricia y Ginecología Vol. 60 No.2 -2009

19. Alix Lozano, Alba Cala, Heidy Molina. "Factores asociados a la no adherencia al programa de control prenatal en las gestantes captadas en el ESE Hospital Sabana de Torres". [Tesis Doctoral] Colombia- Bucaramanga- Santander. Ed 2011.

20. Lina Escobar, Lyda Guzmán, y Ramiro Córdoba. "Factores asociados a la inasistencia al control prenatal durante el primer trimestre en mujeres gestantes de la ESE San Sebastián La Plata Huila 2009". [Tesis Doctoral] NeivaColombia. Ed. 2011. 